\title{
Cyber-physical networking for wireless mesh infrastructures
}

\author{
C. Mannweiler, C. Lottermann, A. Klein, J. Schneider, and H. D. Schotten \\ Chair for Wireless Communications and Navigation, University of Kaiserslautern, Germany \\ Correspondence to: C. Mannweiler (mannweiler@eit.uni-kl.de)
}

\begin{abstract}
This paper presents a novel approach for cyberphysical network control. "Cyber-physical" refers to the inclusion of different parameters and information sources, ranging from physical sensors (e.g. energy, temperature, light) to conventional network information (bandwidth, delay, jitter, etc.) to logical data providers (inference systems, user profiles, spectrum usage databases). For a consistent processing, collected data is represented in a uniform way, analyzed, and provided to dedicated network management functions and network services, both internally and, through an according API, to third party services. Specifically, in this work, we outline the design of sophisticated energy management functionalities for a hybrid wireless mesh network (WLAN for both backhaul traffic and access, GSM for access only), disposing of autonomous energy supply, in this case solar power. Energy consumption is optimized under the presumption of fluctuating power availability and considerable storage constraints, thus influencing, among others, handover and routing decisions. Moreover, advanced situation-aware auto-configuration and self-adaptation mechanisms are introduced for an autonomous operation of the network. The overall objective is to deploy a robust wireless access and backbone infrastructure with minimal operational cost and effective, cyber-physical control mechanisms, especially dedicated for rural or developing regions.
\end{abstract}

\section{Introduction}

Information and communication technology (ICT) infrastructure (both operator equipment and consumer electronics), besides being very costly, is generally developed according to needs and requirements of urban areas in developed countries. In rural areas and even more so in developing countries (except for the big cities), we encounter completely different conditions and deployment environments and significant financial restrictions. Therefore, the most feasible approach is to conceptually and technically adapt, extend, and improve existing equipment so that it better reflects the needs of sparsely populated regions in developing countries. The SolarMesh project, a consortium funded by the German Ministry of Education and Research, consisting of two research institutions and three companies, develops such an autonomous, resource-efficient, easily deployable and maintainable system, as presented in this work. One of the key challenges is to model the infrastructure as a multi-domain system, thus reflecting the dependencies between different parameters and facilitating a network management across different scales (Horstemeyer, 2010).

The remainder of the paper is structured as follows: the next section briefly describes the overall objectives of the SolarMesh project. Section 3 then provides an overview of related work, in particular multi-domain systems and how according concepts can be used to intelligently manage the SolarMesh infrastructure. Section 4 outlines the architectural concept of the considered wireless mesh network (WMN). Key innovations the project addresses are presented in Sect. 5, whereas Sect. 6 discusses expected results. The paper concludes with a summary and description of future work in Sect. 7.

\section{Technical objectives}

The SolarMesh wireless mesh network has been designed for deployment in rural areas of both industrialized and developing countries as a means to provide cost-efficient backhaul connectivity. The key objectives guiding the development, as listed below, have been derived from that major motivation:

1. High-performing, adaptive and extensible system architecture

The overall architecture will be developed in a way that technologies beyond those considered in the project 
(WLAN IEEE 802.11 and GSM) can be integrated easily. This holds particularly true for those alternative radio technologies reducing costs, energy consumption, or access barriers. Therefore, the integration of GSM as an additional access technology is motivated by three reasons: GSM terminals are relatively affordable, their energy consumption is comparably low, and, finally, they are very common in (rural) Africa. Moreover, many services, including governmental services, merely rely on SMS signaling. However, the termination of the GSM connection already takes place in the access points in order to avoid expensive GERAN infrastructure.

2. Development of a mesh network based on autonomous energy supply

Energy supply of the mesh nodes is based on regenerative sources (solar power). Hence, all components (hardware, software, backbone, and air interface) and mechanisms (routing, scheduling, handover, radio transmission) have to be optimized taking into account fluctuating power availability and limited energy storage capacities. The partners will develop according advanced energy-aware methods for network control.

\section{Advanced auto-configuration capabilities}

Deployment and start up of the mesh network will not necessarily require technically trained personnel. Individual nodes as well as the network as a whole will be mostly configured and adapted autonomously to their respective deployment area.

\section{Advanced self-adaptation capabilities}

Besides considering energy efficiency as a main factor, network management and load balancing have to support a large-area mesh network with time-variant topology and dynamically changing traffic load. Availability of individual nodes will be significantly reduced due to limited energy resources, extreme weather conditions (high temperatures, strong rain, sand storms), and maintenance work. The network will have to cope with these characteristics in an autonomous and signaling-efficient manner.

\section{Implementation of efficient handover und routing mechanisms}

Handover and routing processes will be optimized and executed in a situation-aware manner to facilitate mobility support for the user and multi-KPI (key performance indicator) network management for the operator (Mihailovic et al., 2009). For the latter, this allows for both control and prediction of power consumption, radio link availability, load distribution in the network, and cognitive use of available spectrum. The user will benefit from seamless connectivity, independent of service and radio technology.

\section{Related work}

Carrier-grade wireless mesh networks have been subject of former research projects. In EU FP7 CARMEN (Kretschmer and Robitzsch, 2010), for example, a wireless mesh infrastructure has been developed that abstracts from different link layer technologies by the usage of IEEE 802.21. The design of the infrastructure and the routing mechanisms provides support for different QoS classes.

\subsection{Energy models}

Generally, system-level models optimize the overall system performance with respect to a chosen set of parameters (Chan et al., 2006; Schrijver, 1986). When including energy consumption, transmission power is an important factor. Accordingly, current energy models particularly optimize network topology (i.e. the location of access points or base stations). Energy consumption is modeled based on path loss $\left(\frac{1}{d^{n}}\right.$, where $d$ denotes the distance between transmitter and receiver and $n$ represents the path loss exponent that depends on the environments of the considered system), with additional consideration of large-scale fading using log-normal distributions as well as small-scale fading using Rayleigh distributions, e.g. Rodoplu and Meng (1999) and Wieselthier et al. (2000).

\subsection{Energy efficiency metrics}

For deployed nodes of wireless ad-hoc networks running with regenerative energy sources (e.g. solar power for countries of sub-Saharan Africa), routing protocols also play an important role in energy consumption (Chang and Tassiulas, 2000). Several energy-aware routing algorithms have been developed that take the characteristics of regenerative energy supplies and the referring outage situations into consideration. In Badawy et al. (2008), an energy-aware routing within the scope of wireless mesh networks based on a WLAN architecture is mapped to an assignment problem and solved by a generic algorithm. In Serrano et al. (2010), the general relation between energy consumption and throughput in the scope of WLAN infrastructures is given. Frequently, total energy consumption is modeled in a linear way, using metrics for consumed energy per routed information unit, such as "energy per bit" or "energy per packet". Obviously, these linear models do not properly reflect energy consumed due to unsuccessful attempts to access the channel (media contention), messages lost due to collision, bit errors, or loss of wireless connectivity (Feeney and Nilsson, 2001).

\subsection{Multi-domain modeling}

The notion of cyber-physical systems (CPS) has been introduced, among others, by Lee (2008). Cyber-physical systems are characterized by the integration of physical systems and processes with networked computing (Krogh et al., 2008). 


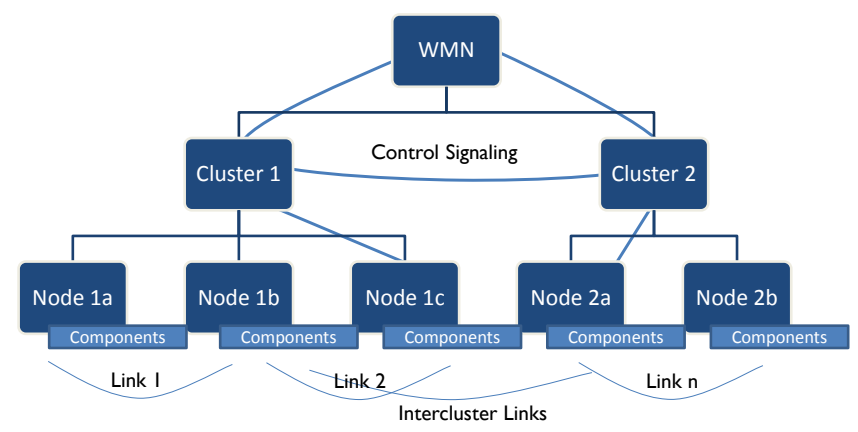

Fig. 1. Hierarchical Energy-Aware Network Management.

The "integration" refers to a tight interaction and mutual dependency of physical resources, such as ICT infrastructures and computational resources, e.g. algorithms to intelligently control these infrastructures. Insofar, numerous systems are categorized as "cyber-physical" or "multi-domain", among them robotic systems, automotive and avionic systems, infrastructures (utilities, communication networks), defense systems, or medical systems. While models describing either domain separately frequently exist, there is a need to design cross-domain models for analyzing and optimizing multi-domain systems. These models better reflect the close interdependency of the physical, computational, and network domain. Within the scope of this paper, the authors present an approach for describing wireless mesh networks as multidomain systems. In particular, energy consumption and efficiency (physical domain) can be modeled depending on, among others, network load and topology (network domain) and user mobility (physical domain). Energy consumption will then be optimized using sophisticated analytical tools (computational domain) that can trigger network management decisions, such as shutting down entire nodes or selected radio interfaces of individual nodes or adapting transmission power levels. Figure 1 depicts the different hierarchical levels on which an energy-aware network management can be executed, ranging from a global view, i.e. the entire network, to components of individual nodes and selected wireless links.

\section{System concept}

With a special focus on the multi-domain modeling challenges, the following section describes the system concept of SolarMesh. It depicts an introduction to the overall system architecture, the auto-configuration and self-adaptation mechanisms, and finally to the handover and routing mechanisms that are implemented.

\subsection{Network architecture}

Several SolarMesh nodes create a wireless, autonomous meshed backhaul network based on IEEE 802.11 wireless technology, as depicted in Fig. 2. The mesh network has three tasks: providing connectivity to the external core network, offering heterogeneous wireless access to user terminals and, most importantly, serving as a wireless backhaul network within a particular area, thus representing an alternative to wired infrastructure. The first one is realized by several gateway connections to the core network and the Internet, whereas the second one is provided by utilizing commonly used Wide Area Network (WAN) technologies, e.g. GSM (in order to take advantage of the cheap off-the-shelf user devices) or Wireless LAN (thus exploiting its affordable network components). Backhaul connectivity is solely based on IEEE 802.11, incorporating mechanisms for carrier-grade QoS, i.e. different services and the accordingly required QoS classes are supported by the overall SolarMesh architecture, namely best-effort and delay-sensitive VoIP services.

The overall system architecture consists of several nodes, disposing of different functionalities:

- SMN (SolarMesh Node) holds the SolarMesh capabilities and embodies a basic interface for other nodes within the architecture. The main task of the SMNs is to build up the mesh backhaul network and to forward the user- and control-plane traffic with respect to the QoS requirements among the SMNs (to the target sink).

- SMGW (SolarMesh Gateway) constitutes the gateway of the wireless backhaul network to the external backbone networks via Global Area Networks (GANs), e.g. a satellite link.

- SMAP (SolarMesh Access Point) provides the wireless access interface to the user terminals. It is equipped with at least one radio interface that does not carry backhaul traffic but is dedicated to the user access. Supported access technologies are IEEE 802.11 and GSM.

- SMC (SolarMesh Coordinator) performs cluster-wide, centralized decisions within a cluster of SMNs. The task of the SMCs is to consider both cluster- and network-wide optimization goals for radio planning, energy-aware node and link configuration, capacity management, or handover decisions.

Besides a comprehensive multi-domain modeling of the system for simulation purpose, a future testbed will be set up with mesh nodes (disposing of at least three 802.11 interfaces) which will be used to deploy the SolarMesh backhaul network. To realize the GSM-based access network, SMAPs will be extended with additional USRP 2 from Ettus LLC controlled by openBTS and an Asterisk PBX software. 

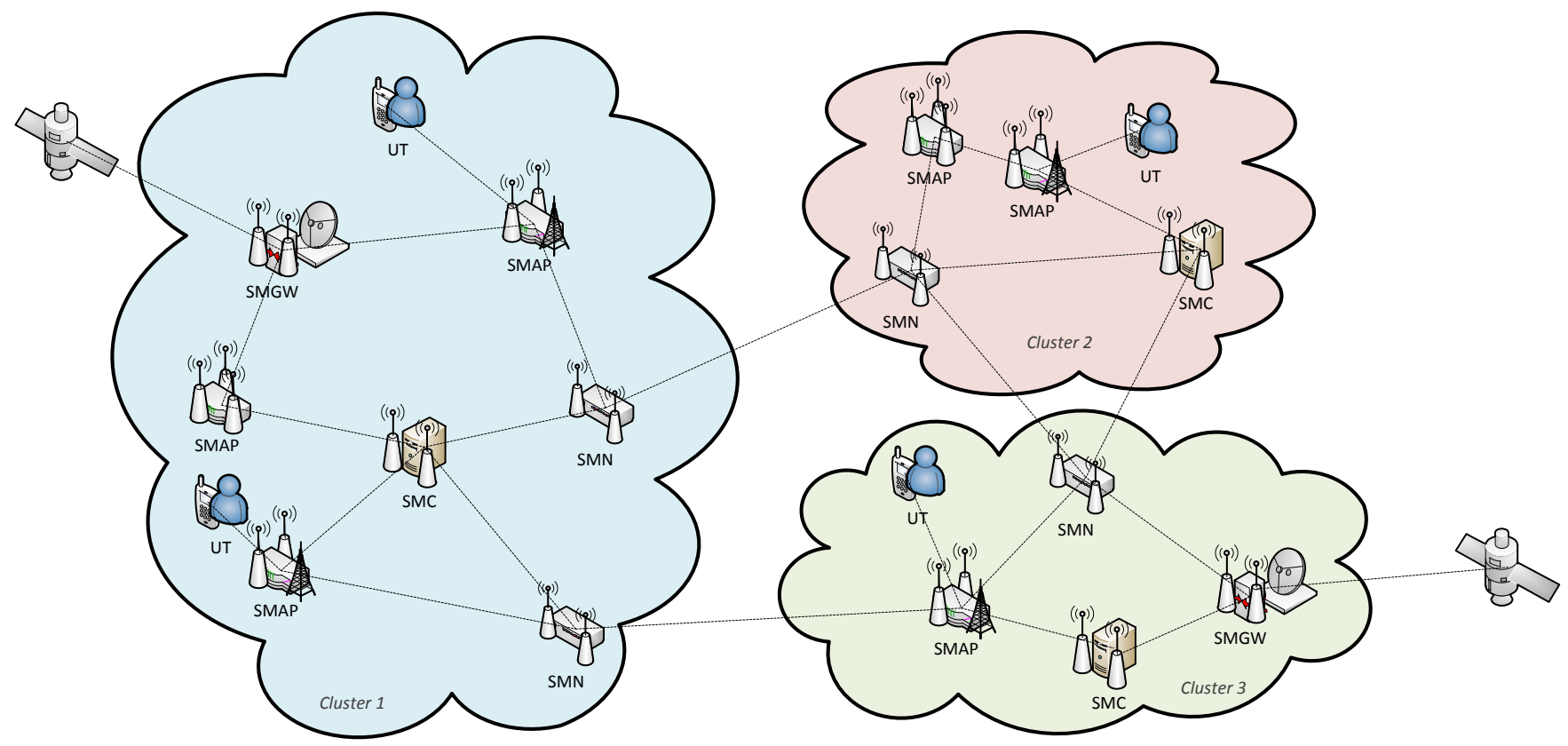

Fig. 2. Overview of System Concept.

\subsection{Multi-domain self-optimization}

One of the main characteristics of SolarMesh is the ability of auto-configuration and self-adaptation, as shown in Fig. 3. This is realized by an autonomous topology detection and configuration of the deployed SMNs. They are grouped into clusters in order to decrease the overall signaling effort and to perform local, i.e. cluster-wide, optimization concerning routing and handover decisions. For these optimization decisions, aspects from the physical domain (e.g. user movements), the computational domain (e.g. energy efficiency statistics), and the network domain (e.g. traffic load) are taken into account. Within a cluster, a SMC is determined that is responsible for the cluster-wide decisions and optimizations. Hence, a SMC can decide to switch off a SMN due to low utilization of the according SMN or its low energy status, e.g. caused by an almost empty buffer battery and, simultaneously, no energy supply by solar panels. As a result, the network topology is reconfigured and the traffic of the considered SMN is dynamically re-routed via other routes. SMNs are able to modify transmission power, modulation and coding schemes, respectively, as well as provided QoS in an autonomous way. Depending on the actual objective function, the emphasis of the optimization can be put on different domains of the system.

For example, target access points for the user terminals are selected depending on the load situation and energy level of the target SMAPs, the capabilities of the user terminals and the SMAPs, and the QoS that has been requested by the user terminal.

\subsection{User mobility and energy-aware routing}

As outlined in previous sections, handover and routing decisions are primarily based on mobility, load situation, and energy situation of the SMNs as well as the provided and requested QoS parameters. Mobility-based handovers are triggered by the local SMNs, whereas the remaining handover decisions are performed by the SMCs in order to allow for a cluster-wide or global optimization of traffic load and energy consumption. Both horizontal, i.e. within the same radio technology, and vertical, i.e. across different radio networks, handovers can be performed. For each end-to-end connection, the most efficient path with respect to energy status or load situation is selected.

\section{Key innovations}

Within SolarMesh, several research challenges need to be tackled which will be described in the following section.

\subsection{Energy awareness and autonomous power supply}

Individual components of the SolarMesh network will be designed to work in regions with specific characteristics: harsh environmental and climatic conditions and unreliable infrastructures, e.g. with respect to energy supply or availability of maintenance resources. These constraints are explicitly considered in the system model as they are typical characteristics of a cyber-physical system. In order to guarantee a continuous operation mode, nodes are equipped with their own energy supply. Power consumption of the SMNs needs 


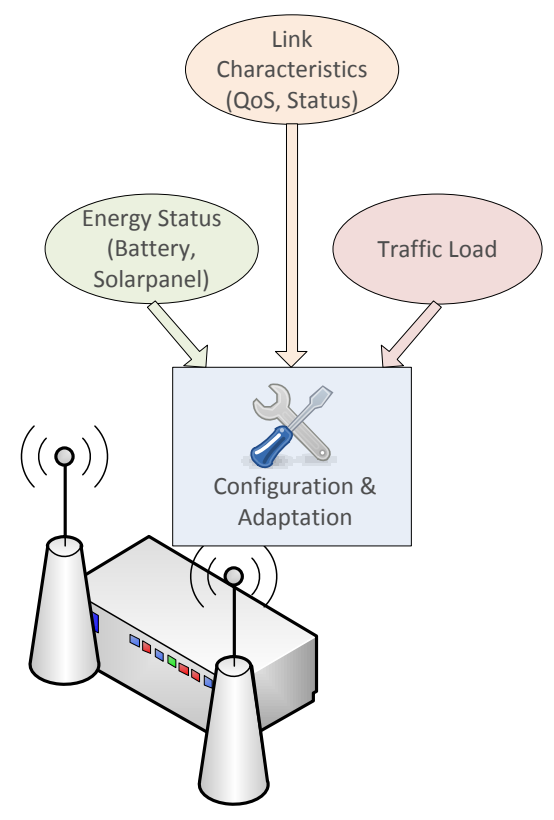

Fig. 3. Auto-Configuration and Self-Adaptation Functionalities of a SMC Node.

to be kept at a minimum aiming at achieving a long operation time. Therefore, an energy management function (EMF) is required that is capable of reducing power consumption by shutting down certain capabilities. This EMF employs a detailed multi-domain energy model of the SMNs. The model uses the power consumption of the supported wireless access networks (e.g. IEEE 802.11 and GSM), depending on different modulation modes, supported states of operation as well as state changes, as input parameters. Additionally, the influence of traffic load on the SMNs is considered, which in turn is dependent on the type of the node: SMCs need to handle more control plane traffic than simple SMNs which in turn leads to a higher power consumption. In general, decisions concerning energy management are taken at different hierarchy levels, as depicted in Fig. 4. As an example, modulation schemes for the terminal link can generally be decided at the local node. In contrast, the decision of shutting down an entire node can only be taken at higher levels (cluster or global), since the individual node is not foreseen to have enough information to take that decisions and to assess the consequences.

\subsection{Network management}

Within the meshed network infrastructure, an advanced network management entity is required in order to perform the required reconfigurations of the constantly changing network topology. This entity has instances at node, cluster, and global level and implements, among others, routing and re-routing mechanisms, handover algorithms, as well as network topology management functionalities. Besides network-side parameters, such as dynamic characteristics of

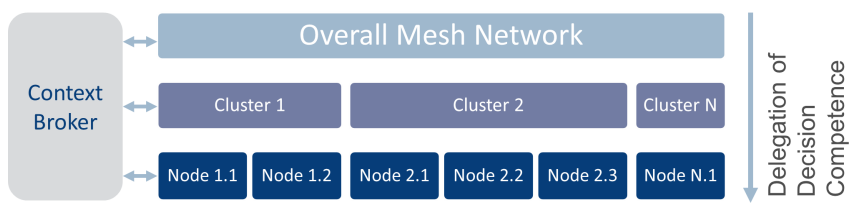

Fig. 4. Network Energy Management Concept.

load or traffic situation with respect to QoS classes, it takes into consideration mobility and capabilities of registered user terminals (such as supported air interfaces, display size, or available codecs), thus also reflecting relevant input parameters from the physical domain.

\section{Expected results}

Within SolarMesh, a wireless mesh infrastructure will be realized that keeps the overall deployment and operational costs at a very low level, as required for operation in rural areas of sub-Saharan Africa. From a theoretical perspective, multi-domain modeling concepts (such as cyberphysical systems) are expected to facilitate the according design of the mesh network and to fulfill these objectives. The overall system design will be evaluated by a mixed simulation and emulation and will finally be implemented and validated in a testbed environment. Routing and handover decisions will be optimized and accelerated by the usage of context-aware decision functions. Seamless connectivity will be realized by the support of mobility (horizontal and vertical handover) and dynamic routing and re-routing. Due to the usage of advanced energy management functions, a significant reduction of the overall power consumption will be realized. Most importantly, the mesh network will rely on and being able to cope with autonomous energy supply solutions, thus gaining independence from locally deployed, potentially unreliable energy grids.

\section{Conclusion and outlook}

The authors have presented how analytical concepts of cyberphysical systems can be used for an advanced management of a wireless mesh network. In particular, the exploitation of information that is available from different domains (physical, computational, network) supports the design of a multidomain system model. Moreover, motivation, objectives, research challenges, and the initial system concept of the SolarMesh project have been outlined. Deployment scenarios, such as rural areas in both developed and developing countries, have been described.

Applying multi-domain models to wireless mesh networks is particularly useful for improving energy efficiency that depends on factors from different domains, such as, among 
others, traffic load in the network (utilization), amount and mobility of users, network and environment topology, transmission power levels, and employed radio technologies. Analyzing and mathematically describing the dependencies between these quantities facilitates the optimization of the network with regards to selected parameters, e.g. energy consumption.

In a first step, future work therefore includes the detailed specification and development of a system architecture disposing of hardware and software components that meet the defined requirements. In a second step, the system will be validated and evaluated based on simulations and testbed experiments. Here, the developed multi-domain models will be used to generate quantitative results with respect to the applicability of the selected parameters for optimized management of the wireless mesh network.

Acknowledgements. This work has been funded by the Federal Ministry of Education and Research of the Federal Republic of Germany (Förderkennzeichen 01 BU 1116, SolarMeshEnergieeffizientes, autonomes großflächiges Sprach- und Datenfunknetz mit flacher IP-Architektur). The authors alone are responsible for the content of the paper.

\section{References}

Badawy, G. H., Sayegh, A. A., and Todd, T. D.: Energy Aware Provisioning in Solar Powered WLAN Mesh Networks. Proceedings of 17th International Conference on Computer Communications and Networks (ICCCN), St. Thomas, U.S. Virgin Islands, 2008.

Chan, T. F., Cong, J., Shinnerl, J. R., Sze, K., Xie, M., and Zhang, Y.: Multiscale Optimization in VLSI Physical Design Automation, in: Multiscale optimization methods and applications, edited by: Hager, W. W., Huang, S.-J., Pardalos, P. M., and Prokopyev, O. A., Springer, 2006.

Chang, J.-H. and Tassiulas, R.: Energy Conserving Routing in Wireless Ad-hoc Networks. Proceedings of the IEEE International Conference on Computer Communications (INFOCOM), Tel Aviv, Israel, 2000.
Feeney, L. M. and Nilsson, M.: Investigating the Energy Consumption of a Wireless Network Interface in an Ad Hoc Networking Environment. Proceedings of the IEEE International Conference on Computer Communications (INFOCOM), Anchorage, USA, 2001.

Horstemeyer, M. F.: Multiscale Modeling: A Review, in: Practical Aspects of Computational Chemistry, edited by: Leszczynski, J. and Shukla, M. K., Springer, 2010.

Kretschmer, M. and Robitzsch, S.: Wireless Mesh Network Coverage with QoS Differentiation for Rural Areas. Proceedings of the First International Workshop on Wireless Broadband Access for Communities and Rural Developing Regions (WIRELESS4D), Karlstad, Sweden, 2010.

Krogh, B. H., Lee, E., Lee, I., Mok, A., Pappas, G., Rajkumar, R., Sha, L. R., Vincentelli, A. S., Shin, K., Stankovic, J., Sztipanovits, J., Wolf, W., and Zhao, W.: Cyber-Physical Systems Executive Summary, CPS Steering Group, 2008.

Lee, E. A.: Cyber Physical Systems: Design Challenges. Proceedings of the IEEE International Symposium on Object-Oriented Real-Time Distributed Computing, Orlando, USA, 2008.

Mihailovic, A., Chochliouros, I. P., Georgiadou, E., Spiliopoulou, A. S., Sfakianakis, E., Belesioti, M., Nguengang, G., Borgel, J., and Alonistioti, N.: Situation awareness mechanisms for cognitive networks. Proceedings of the International Conference on Ultra-Modern Telecommunications (ICUMT), St. Petersburg, Russia, 2009.

Rodoplu, V. and Meng, T. H.: Minimum energy mobile wireless networks, IEEE J. Sel. Area. Comm., 17, 1333-1344, 1999.

Schrijver, A.: Theory of Linear and Integer Programming, John Wiley \& Sons, 1986.

Serrano, P., Hollick, M., and Banchs, A.: On the Trade-Off between Throughput Maximization and Energy Consumption Minimization in IEEE 802.11 WLANs, J. Commun. Netw., 12, 19765541, 1229-2370, 150-157, 2010.

Wieselthier, J. E., Nguyen, G. D., and Ephremides, A.: On the Construction of Energy-Efficient Broadcast and Multicast Trees in Wireless Networks. Proceedings of the IEEE International Conference on Computer Communications (INFOCOM), Tel Aviv, Israel, 2000. 